\title{
Enterococcus gallinarum Strain MRx0518
}

National Cancer Institute

\section{Source}

National Cancer Institute. Enterococcus gallinarum Strain MRX0518. NCI Thesaurus.

Code C160293.

A live strain of the flagellin-producing Gram-positive bacterium Enterococcus (E.)

gallinarum that is isolated from a healthy human gut, with potential immunomodulating and antineoplastic activities. Upon oral administration, MRx0518 modulates the intestinal microbiota and targets both intestinal epithelial cells (IECs), and various immune cells, such as macrophages and dendritic cells (DCs) and is able to induce the production of both pro- and anti-inflammatory mediators, such as interleukin-8 (IL-8), tumor necrosis factor-alpha (TNF-a), IL-1 beta, IL-6, IL-23, in these cells and activates the innate immune system. The flagellin produced by MRx0518 interacts with and activates toll-like receptor 5 (TLR5), thereby activating the adaptive immune system and modulating the tumor microenvironment (TME). This activates the immune system to mount a cytotoxic Tlymphocyte (CTL) immune response against tumor cells. 